\title{
CRISP(R)ation musculaire
}

Océane Ballouhey ${ }^{1}$, Marc Bartoli ${ }^{1}$, Nicolas Levy ${ }^{1-3}$

> Les dystrophies musculaires sont un ensemble de pathologies musculaires rares, caractérisées par une faiblesse et une dégénérescence progressive du muscle. Ce sont des maladies d'origine génétique causées par la mutation d'un ou de plusieurs gènes impliqués dans les fonctions musculaires. Malgré des progrès significatifs réalisés dans le champ des biothérapies au cours des dernières années, il n'existe pas, à ce jour, de traitement curatif disponible pour ces pathologies. Les études menées depuis la découverte de l'outil d'édition génomique CRISPR-Cas9 ont néanmoins permis des avancées significatives et prometteuses dans le traitement des dystrophies musculaires. Le système CRISPR-Cas9 permet une édition stable et permanente du génome et doit permettre d'éviter les traitements longs et répétitifs. Dans cette revue, nous aborderons les dernières avancées thérapeutiques utilisant le système CRISPR-Cas9 dans le cadre des dystrophies musculaires d’origine génétique. <

Le muscle squelettique représente environ $40 \%$ de la masse corporelle d'un individu [1]. L'intégrité de ce tissu dynamique et plastique dépend de l'équilibre établi entre la synthèse et la dégradation des protéines qui le composent. Des facteurs, tels que la nutrition, l'équilibre hormonal, l'activité physique, le stress, mais aussi de nombreuses mutations géniques fragilisent I'homéostasie du muscle.

Il existe plus de 800 pathologies monogéniques associées à une dégénérescence et une dysfonction du muscle. Parmi celles-ci, les dystrophies musculaires sont un ensemble de pathologies musculaires rares caractérisées par une faiblesse et une dégénérescence progressive du muscle: les fibres musculaires dégénèrent, les muscles s'atrophient progressivement et une faiblesse musculaire s'installe [2]. Ces pathologies

Vignette (Photo @ Christophe Praud-Inserm/INRA).

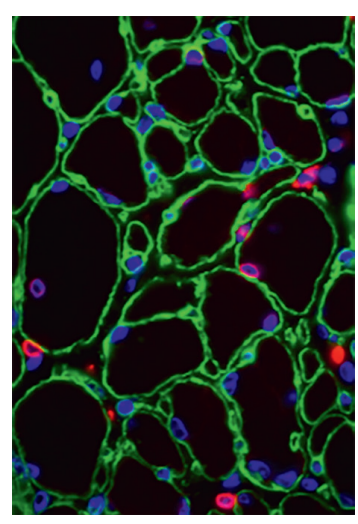

${ }^{1}$ Aix Marseille Univ, Inserm, MMG, U1251, 13005 Marseille, France. ${ }^{2}$ AP-HM Département de Génétique Médicale, Hôpital d'Enfants de la Timone, Marseille, 13005 France. ${ }^{3}$ GIPTIS, Genetics Institute for Patients Therapies Innovation and Science, 13002 Marseille, France.

nicolas.levy@univ-amu.fr

apparaissent à tout âge : rarement dès la naissance, mais le plus souvent durant l'enfance ou l'adolescence, ou encore à l'âge adulte. Ce sont des maladies d'origine génétique causées par la mutation d'un ou de plusieurs gènes impliqués dans les fonctions musculaires, tels que DMD (codant la dystrophine) impliqué dans la dystrophie musculaire de Duchenne (DMD), la dystrophie musculaire la plus connue et la plus fréquente, CAPN3 (codant la calpaïne 3) impliqué dans les dystrophies musculaires des ceintures, LGMD RI et LGMD D4 (limb girdle muscular dystrophy type RI and D4), ou encore DYSF (codant la dysferline) impliqué dans les dysferlinopathies (LGMD R2 et myopathie de Miyoshi).

II n'existe pas de traitement curatif pour les dystrophies musculaires. Cependant, l'identification des gènes responsables de chaque type de dystrophie musculaire et la compréhension des mécanismes qui la soustendent, ont permis des avancées considérables sur le plan du développement d'approches thérapeutiques. Parmi ces dernières, les principales voies explorées sont le saut d'exon utilisant des oligonucléotides antisens (AON), la trans-lecture de codons stop fondée sur des molécules pharmacologiques (gentamycine, TRANSLARNA ${ }^{\circledR}$ ou ataluren) ou encore la thérapie génique par transfert de gène corrigé [3]. L'enjeu de ces stratégies thérapeutiques est d'atteindre les cellules satellites musculaires, cellules souches adultes du muscle strié squelettique logeant le long des myofibres, puisque les principales cellules qui composent le muscle sont des cellules post-mitotiques. La réparation/régénération du muscle est donc assurée par les cellules satellites. Dans le but de mettre en place une thérapie à long terme, il est nécessaire de cibler ces cellules, surtout lorsque le traitement est initié très tôt chez les enfants en pleine croissance.

Ces dernières années, un outil d'édition génomique a permis des avancées majeures dans les approches thérapeutiques pour les dystrophies musculaires, et semble extrêmement prometteur pour devenir un outil de premier plan dans l'arsenal thérapeutique pour les maladies génétiques. Ce système CRISPR-Cas9 permet une édition stable et permanente du génome, et doit permettre d'éviter les traitements longs et 

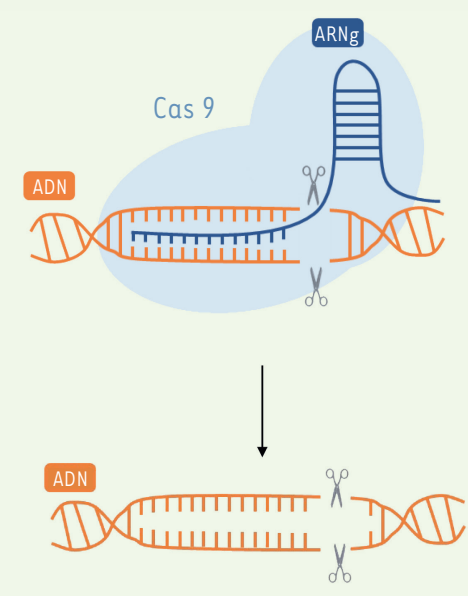

Coupure double brin de l'ADN

Réparation NHEJ

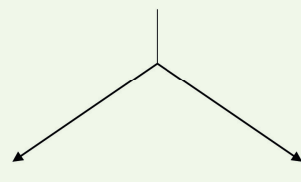

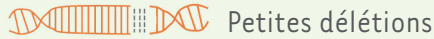

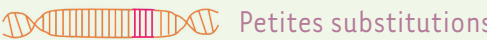

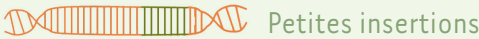

Figure 1. Mécanismes d'action du système CRISPR-Cas9. NHEJ : non homologous end joining ; HDR : homology directed repair.

répétitifs. Les stratégies d'utilisation de cet outil d'édition génomique sont variées. Dans cette revue, nous aborderons les dernières avancées thérapeutiques utilisant le système CRISPR-Cas9 dans le cadre des dystrophies musculaires d'origine génétique.

\section{Le système CRISPR}

Des outils d'édition génomique existent depuis les années 1970 [53]. Ils permettent de modifier précisément une séquence génomique. Les principaux sont les nucléases à doigts de zinc (zinc finger nucleases, ZFN), les méganucléases, les nucléases effectrices de type activateur de transcription (transcription activator-like effector nucleases, TALEN) ou encore, identifié plus récemment, le système CRISPR (clustered regularly interspaced short palindromic repeats)-Cas9 (CRISPR-associated protein 9) [4]. Ce dernier a permis des avancées considérables grâce à sa rapidité d'exécution, à sa facilité d'utilisation, et à son efficacité démontrée dans des modèles cellulaires et vivants utilisés en laboratoire. CRISPR-Cas9 est composé d'une enzyme, Cas9, et d'un ARN guide synthétique $(A R N g)$ qui forment ensemble un complexe ribonucléoprotéique [5]. L'enzyme Cas9 chargée avec I'ARN guide reconnaît une séquence complémentaire située à côté d'une séquence spécifique de 3 à 5 nucléotides (séquence PAM, protospacer adjacent motif). Lorsque le complexe Cas9-ARNg reconnaît un site cible, il déclenche la dénatu- ration locale de I'ADN et l'invasion dans I'ADN du brin d'ARN pour assurer le clivage de la double hélice d'ADN. La coupure double brin créée par l'enzyme Cas9 est alors réparée soit par jonction d'extrémités non-homologues (NHEJ pour non homologous end joining), résultant en de petites insertions et/ou délétions aléatoires (indel) sur le site de clivage, soit par réparation fondée sur l'homologie (HDR pour homology-directed repair), réparation dirigée qui entraîne une modification précise du génome (Figure 1) [6]. Cependant, la précision et l'efficacité de ce système sont modulées par un inconvénient qu'il conviendra de surmonter dans l'avenir. En effet, des éditions génomiques sont susceptibles d'apparaître dans des sites adjacents d'une séquence PAM et qui ne diffèrent que de quelques nucléotides par rapport à la séquence ciblée : ces modifications sont appelées mutations hors cible (off-target) $[7,8]$. Le système CRISPRCas9 est ainsi capable de cliver une séquence d'ADN contenant jusqu'à 5 nucléotides qui diffèrent de l'ARN guide, ce qui est la cause de mutations hors cible. Plus le nombre de mésappariements tolérés par l'outil d'édition génomique est important, plus ce dernier générera des mutations hors cible (Figure 2).

Ces dernières années, le système CRISPR-Cas9 a largement été utilisé dans diverses études. Les possibilités d'utilisation de cet outil sont diverses. L'utilisation de l'enzyme Cas9 et de deux ARN guides peut permettre de déléter un fragment d'ADN, voire même un gène entier [9]. D'un autre côté, lorsque la réparation du clivage induit par Cas9 est réalisée par le mécanisme NHE), il est possible d'altérer le cadre de lecture d'une protéine ou un site d'épissage. Enfin, lorsque la réparation du clivage induit par Cas9 utilise le mécanisme HDR grâce à la présence d'un ADN donneur, il est possible d'insérer un fragment d'ADN, de modifier une séquence afin de créer une mutation, ou encore de corriger une mutation. Ces utilisations diverses peuvent permettre la création de modèles dans différents organismes afin de modéliser une pathologie. Elles permettent également de neutraliser une mutation existante afin de restaurer la production ou la fonction d'une protéine $[10,11]$.

Pour délivrer cet outil d'édition génomique dans les organes cibles, de nombreuses méthodes ont été utilisées. La méthode de transport la plus utilisée in vitro est la transfection d'ADN plasmidique. In vivo, certaines études utilisent des méthodes de transport non-viral pour acheminer le système CRISPR-Cas9, telles que le transport hydrodynamique ou encore le transport par nanoparticules [12-13]. Ces stratégies de transport non-viral permettent une expression transitoire de la protéine Cas 9 et ainsi de réduire les mutations hors cible. La méthode la plus utilisée pour transporter le 


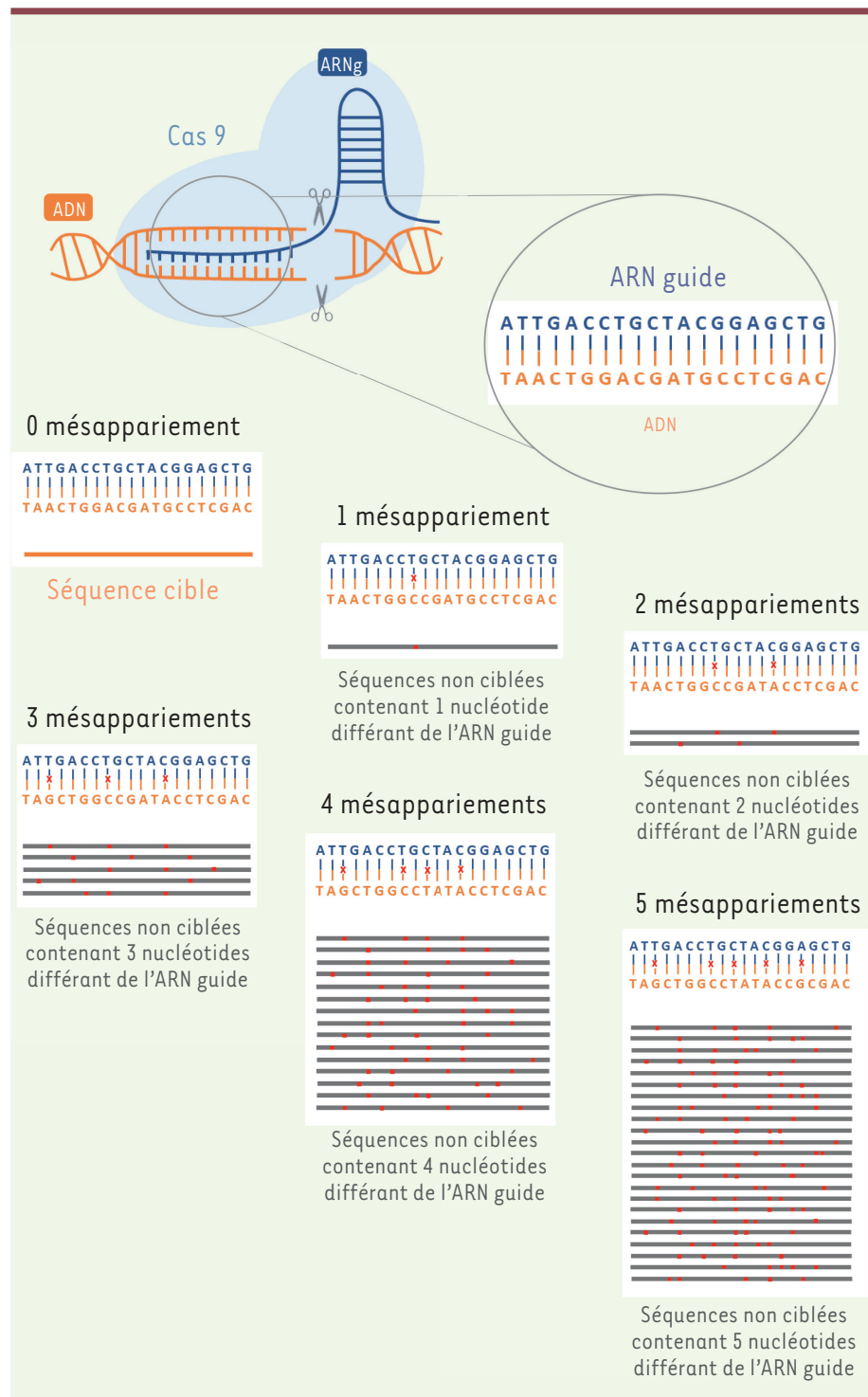

Figure 2. Le système CRISPR-Cas9 peut causer des mutations hors cible puisqu'il est capable de cliver une séquence d'ADN contenant jusqu'à 5 nucléotides différant de la séquence de l'ARN guide.

système CRISPR-Cas9 in vivo reste le transport par AAV (virus associés aux adénovirus) [14]. Ce système viral très efficace dans le muscle et versatile est fortement utilisé en thérapie génique, malgré sa capacité d'encapsidation assez faible.

\section{Création d'organismes modèles}

Dans le cadre des dystrophies musculaires, de nombreux modèles ont été créés en utilisant le système CRISPR-Cas9, notamment pour la myopathie de Duchenne causée par des mutations dans le gène DMD. Nakamura et son équipe ont ainsi généré un rat afin de modéliser cette pathologie [15]. Pour cela, deux exons du gène Dmd ont été ciblés dans le but de les éliminer et ainsi empêcher la production de dystrophine. Grâce à cette modification génomique ciblée, ce rat pré- sente de nombreuses caractéristiques phénotypiques et mécanistiques de la pathologie, avec notamment une diminution de la force musculaire et l'émergence de phénotypes histologiques dégénératifs/régénératifs dans le muscle squelettique, le cœur et le diaphragme. II représente ainsi un excellent modèle de DMD. Plus récemment, en 2018, deux équipes ont également utilisé le système CRISPR-Cas9 afin d'obtenir des organismes modèles de DMD: une souris dont l'exon 50 du gène Dmd a été délétée [16], et un lapin possédant des mutations dans l'exon 51 du même gène [17]. Enfin, un modèle de la myopathie a été obtenu grâce à l'outil CRISPR-Cas 9 chez le singe en ciblant les exons 4 et 46 du gène $D m d$, afin d'en faire un modèle pertinent d'évaluation des stratégies thérapeutiques [18].

D'autres modèles de dystrophies musculaires ont également été créés. Une souris modélisant la dystrophie musculaire des ceintures de type R5 (LGMD R5) a ainsi été obtenue par Demonbreun et ses collaborateurs [19]. Cette pathologie est causée par des mutations dans le gène SGCG qui code la $\gamma$-sarcoglycane. Dans le but de reproduire la mutation principale de cette dystrophie musculaire $(521 \Delta \mathrm{T})$, les auteurs ont utilisé le système CRISPR-Cas9 afin de déléter un nucléotide de l'exon 6 du gène SGCG, produisant un décalage du cadre de lecture et donc l'absence de production de la protéine. Une autre souris modélisant la myopathie liée au récepteur RYRI (ryanodine receptor 1 ) a été réalisée [20] par Brennan et ses collaborateurs qui ont introduit une mutation ponctuelle identique à celle identifiée chez un patient, dans un allèle du gène Ryrl, ainsi qu'une délétion de 16 nucléotides produisant un décalage du cadre de lecture dans le deuxième allèle. Ce modèle murin présente une réduction de la masse musculaire due à une atrophie des myofibres, avec un impact sur la masse corporelle globale. L'outil CRISPR-Cas9 a donc permis, ces dernières années, de modéliser plusieurs formes de dystrophies musculaires, de façon plus précise, rapide et efficace que les approches utilisant la réparation par homologie permettant l'inactivation d'un gène (knock-out).

\section{Thérapie par saut d'exon}

Le système CRISPR-Cas9 ne permet pas seulement de modéliser une pathologie, il rend possible son traitement. Une des utilisations thérapeutiques du système CRISPR-Cas9 est l'altération d'un site d'épissage afin de réaliser un saut d'exon pour restaurer le cadre de lecture permettant de produire une protéine (Figure 3). À la différence de la thérapie par saut d'exon utilisant des oligonucléotides antisens (AON), l'utilisation de 


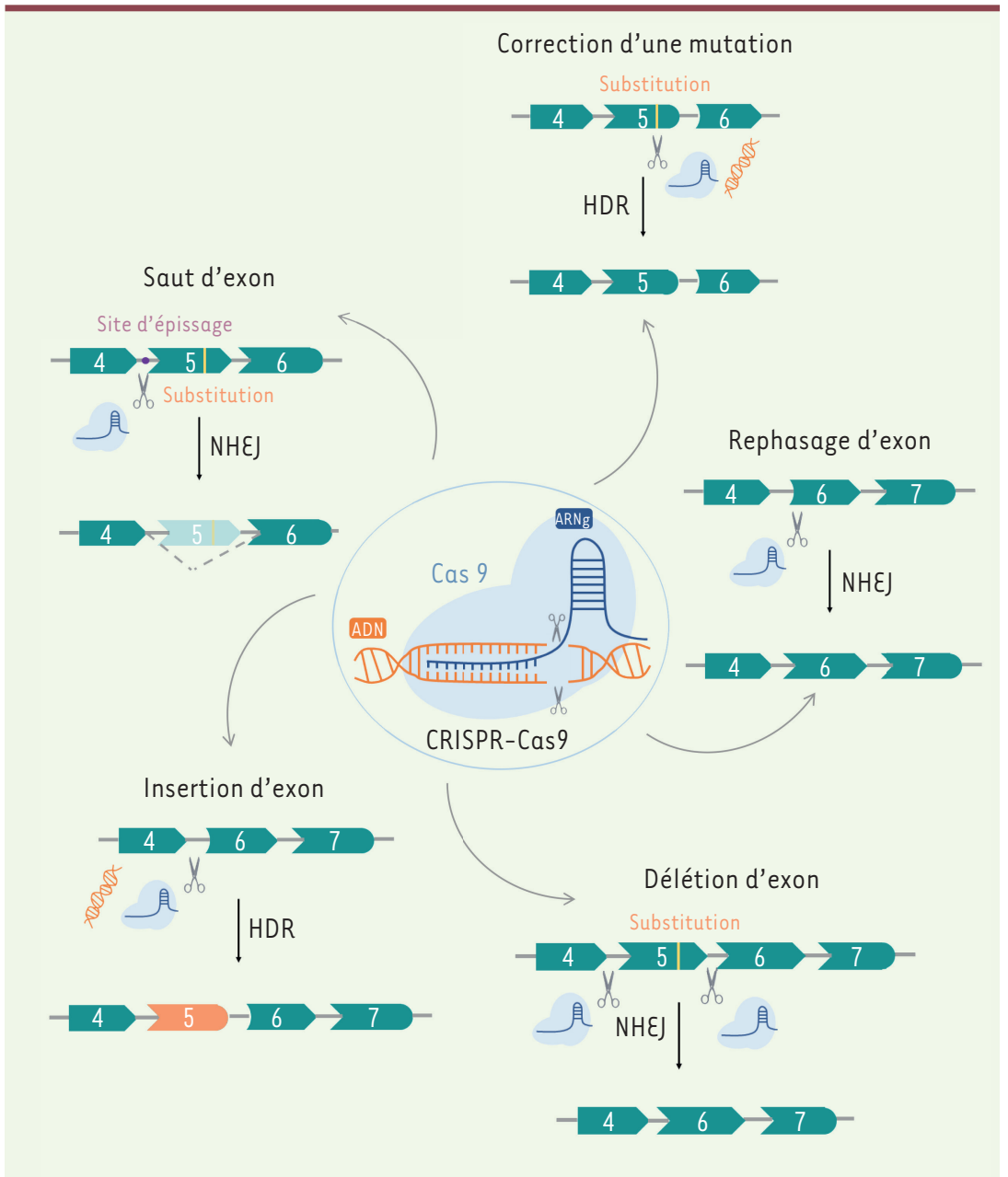

Figure 3. Stratégies thérapeutiques utilisant le système CRISPR-Cas9.

CRISPR-Cas9 permet d'altérer un site d'épissage afin d'empêcher l'incorporation d'un exon dans l'ARN messager (ARNm) mature par le spliceosome. Cette approche a été largement utilisée ces dernières années, particulièrement pour en évaluer la pertinence et l'efficacité dans la DMD. Dans ce contexte, les chercheurs ont ciblé une région correspondant à un site fréquent de mutations du gène $D M D$ (exons 45 à 55). Les mutations dans cette région provoquent un décalage du cadre de lecture, constituant ainsi un fort rationnel pour l'exploration du saut d'exon dans cette dystrophie musculaire.

Ainsi, Amoasii et son équipe ont utilisé l'outil CRISPR-Cas9 dans le but de réaliser un saut de l'exon 51 du gène $D M D$ dans des modèles de mammifères présentant une délétion de l'exon 50 : la souris $\Delta \varepsilon \times 50$ [16], la souris $\Delta \varepsilon \times 50-D m d-L u c$ [21] et le chien $\Delta \varepsilon \times 50-M D$ [22]. La délétion de l'exon 50 du gène $D m d$ provoque une altération du cadre de lecture, menant alors à l'absence de production de la dystrophine. Le saut de l'exon 51 permet alors la restauration du cadre de lecture et la production d'une protéine plus courte mais fonctionnelle. Ces 3 études thérapeutiques ont conduit à des résultats encourageants: restauration de la dystrophine dans le muscle squelettique, le muscle cardiaque et le diaphragme.
Suivant le même principe, l'équipe de Long, en 2016, a utilisé CRISPR-Cas9 chez les souris $m d x$, modèle standard de DMD, qui présentent une anomalie génétique dans l'exon 23 de la dystrophine, afin de réaliser le saut de l'exon 23 du gène $D m d$ [23]. Pour cela, ils ont délivré le système CRISPR-Cas9 dans le muscle ciblé grâce au virus AAV9. Leurs résultats indiquent une restauration de la dystrophine dans les muscles cardiaque et squelettique, ainsi qu'une amélioration des fonctions musculaires squelettiques (force de préhension). $\varepsilon n 2018$, Ifuku et ses collaborateurs ont altéré le site accepteur d'épissage de l'exon 45 du gène DMD afin de provoquer le saut du même exon dans des cellules souches pluripotentes induites (iPS) dérivées d'un patient atteint de DMD dépourvu de l'exon 44 du gène DMD [24]. Ceci a permis de restaurer le cadre de lecture et ainsi l'expression de la dystrophine dans des myoblastes différenciés. Enfin, l'équipe de Long, toujours en pointe, a utilisé des ARN guides permettant d'introduire des petites insertions/délétions (indel) au niveau de 12 sites d'épissage dans des lignées d'iPS de patients atteints de DMD afin de provoquer le saut des exons correspondants, qui sont fréquemment mutés dans le gène $D M D$ [25]. Cette étude a permis de restaurer l'expression de la dystrophine dans les cardiomyocytes dérivés d'iPS et de démontrer que la correction de 30 à $50 \%$ des cardiomyocytes était suffisante pour restaurer le phénotype cardiaque à un niveau proche de la normale.

\section{Thérapie par délétion d'exons}

L'outil CRISPR-Cas9 a également été utilisé afin de déléter un ou plusieurs exons, dans le but de restaurer la cadre de lecture d'un gène ou de supprimer un ou plusieurs exons contenant des mutations (Figure 3 ). À la différence de la thérapie par saut d'exon, cette approche thérapeutique n'implique pas la machinerie de l'épissage. Cette utilisation thérapeutique de CRISPR-Cas9 a été explorée dans le cadre des dystrophies musculaires. Duchêne et ses collaborateurs ont ainsi restauré le cadre de lecture de la dystrophine à partir de quatre biopsies musculaires de patients atteint de DMD (chaque biopsie possédant une délétion d'un exon différent) [26]. Pour cela, ces auteurs ont utilisé l'enzyme Cas9 et deux ARN guides ciblant chacun 
l'exon 47 et l'exon 58 du gène DMD afin de déléter les exons 48 à 57 et ainsi former un exon chimérique avec la partie 5' de l'exon 47 et la partie 3' de l'exon 58 du gène. Cette technique a permis de restaurer l'expression d'une forme plus courte de dystrophine fonctionnelle dans les cellules des quatre patients.

L'enzyme Cas9 et deux ARN guides ciblant les introns 22 et 23 du gène Dmd ont par ailleurs été utilisés chez des souris $m d x$ [27-29]. Cette méthode a permis d'éliminer la mutation présente dans le gène $D m d$ des souris $m d x$ grâce à la délétion de l'exon 23, et l'expression de la dystrophine a pu être restaurée dans les myofibres, les cardiomyocytes et les cellules souches musculaires. L'expression de la protéine a persisté durant 18 mois, et les fonctions musculaires ont été partiellement restaurées.

Dans le cadre de la dystrophie myotonique de type 1 (DMl ou maladie de Steinert), deux équipes ont utilisé le système CRISPR-Cas9 afin de déléter la répétition CTG, présente dans la région 3' du gène DMPK (dystrophia myotonica l) et à l'origine de la pathologie [30-31]. Lorsque ce triplet CTG est répété de 50 à plusieurs milliers de fois, le fonctionnement des muscles est atteint, ainsi que celui d'autres organes, selon la forme de la maladie (difficultés respiratoires, troubles cardiaques, troubles hormonaux, etc.). Dastidar et al. ont utilisé CRISPR-Cas9 afin d'éliminer ces répétitions CTG grâce à deux ARN guides dans des cellules myogéniques dérivées d'iPS de patients présentant une DMI [30]. Leur étude montre la disparition des foyers ribonucléaires et la restauration de la localisation de MBLNI (muscleblind like splicing regulator 1 ), un régulateur d'épissage alternatif autorégulant la transcription du gène DMPK dans les cellules myogéniques. Une étude réalisée sur des cellules musculaires dérivées d’iPS de patients atteints de DMl, et chez une souris modélisant la DMI (la souris transgénique DMSXL ${ }^{1}$ ) montre qu'avec cette méthode, les foyers de ribonucléoprotéine disparaissent et que les anomalies d'épissage dans divers transcrits sont corrigés dans les cellules musculaires dérivées d'iPS des patients et chez la souris [31].

\section{Thérapie par correction de mutations}

Le système CRISPR-Cas9 permet aussi de corriger des mutations grâce à la présence d'un ADN donneur afin de restaurer l'intégralité d'un gène par HDR (Figure 3). Cette stratégie thérapeutique a été explorée dans plusieurs dystrophies musculaires. Selvaraj et ses collaborateurs ont, par exemple, restauré la protéine calpaïne 3 , codée par le gène CAPN3, dans des myotubes dérivés de trois lignées iPS de patients atteints de dystrophie musculaire des ceintures de type R1 [32]. Pour cela, ils ont utilisé l'enzyme Cas9, un ARN guide ciblant la mutation présente dans le gène CAPN3 et un ADN donneur afin de réparer par HDR, le clivage induit par Cas9 dans ces cellules iPS mutées. Ils ont ensuite transplanté les progéniteurs myogéniques corrigés dans un modèle murin présentant un déficit immunitaire et en calpaïne 3. Cette étude a révélé le potentiel de CRISPRCas9 pour la thérapie génique couplée à la thérapie cellulaire. Le système

${ }^{1}$ Souris porteuses de grandes séquences génomiques humaines $(45 \mathrm{~kb})$ contenant le gène DMPK et une importante expansion de triplets répétés CTG.
CRISPR-Cas9 et un ADN donneur ont également été utilisés par Wang et son équipe, dans le but d'éliminer les répétitions toxiques du triplet CTG dans la région 3' du gène DMPK dans des lignées iPS de patients DMl. Pour cela, des signaux de polyadénylation ont été insérés en amont des répétitions CTG afin d'achever prématurément la transcription. Cette stratégie thérapeutique a permis d'augmenter la précision de correction par rapport à l'approche de délétion des répétitions CTG avec pour résultats, la réversion du phénotype dans les cellules souches neurales, les neurones du cerveau antérieur, les cardiomyocytes et les myofibres du muscle squelettique [33].

De nouveau dans le cadre de la DMD, plusieurs études montrent la correction des mutations dans le gène $D M D$ grâce au système CRISPR-Cas9 et la présence d'un ADN donneur [34-36]. Long et al. ont ainsi utilisé cette méthode afin de corriger la mutation présente chez la souris $m d x$ dans sa lignée germinale [34]. L'édition génomique de ces souris a conduit à la production d'animaux mosaïques présentant entre 2 et $100 \%$ de correction du gène $D m d$, avec une restauration partielle de la dystrophine. Une construction Cas9 muscle-spécifique, contenant l'ARN guide et une région homologue à la dystrophine (ADN donneur), a également été utilisée pour corriger la mutation de la souris $m d x$ [35]. Les muscles traités expriment alors des taux de dystrophine atteignant $70 \%$ du taux normal, et recouvrent une force importante après l'injection intramusculaire du système CRISPR-Cas9. Son injection dans la circulation induit l'expression généralisée de la dystrophine dans les muscles squelettiques et cardiaques. L'utilisation d'une construction musclespécifique permet de limiter le risque de mutations hors cible dans les cellules non-musculaires et de minimiser le déclenchement d'une réponse immunitaire.

Matre et al. ont, eux, obtenu la restauration de la dystrophine en ciblant l'exon 23 du gène Dmd dans des progéniteurs musculaires de souris $m d x$ [36] avec pour résultats encourageants, I'amélioration de la prolifération et de la différenciation cellulaire, et la résistance des cellules au stress oxydatif et au stress du réticulum endoplasmique. De même, en utilisant CRISPR-Cas9 et un $A D N$ donneur afin d'induire une réparation par homologie (HDR), Lee et al. ont réussi à corriger la mutation du gène $D m d$ chez la souris $m d x$ [11] avec une restauration de l'expression de la dystrophine dans les tissus musculaires. Ces auteurs ont utilisé des nanoparticules d'or afin de transporter directement la protéine Cas9 dans les cellules, et obtenir un clivage rapide de I'ADN et réduire les mutations hors cible.

Ces stratégies thérapeutiques, qui ont en commun l'utilisation d'un ADN donneur, présentent des résultats prometteurs pour le traitement des dystrophies musculaires. 


\begin{tabular}{|c|c|c|c|c|c|}
\hline Pathologie & Modèle & Enzyme Cas9 & Transport & Stratégie & Références \\
\hline \multirow{13}{*}{$\begin{array}{l}\text { Dystrophie } \\
\text { musculaire de } \\
\text { Duchenne } \\
\text { (DMD) }\end{array}$} & Souris $\Delta \varepsilon \times 50$ & \multirow{3}{*}{ SpCas9 } & \multirow{3}{*}{ AAV9 (IM) } & \multirow{3}{*}{ Saut de l'exon 51 du gène $D M D$} & [12] \\
\hline & $\begin{array}{l}\text { Souris } \\
\Delta \varepsilon \times 50-\text { Dmd-Luc }\end{array}$ & & & & [17] \\
\hline & Chien $\Delta \varepsilon \times 50-M D$ & & & & [18] \\
\hline & \multirow{6}{*}{ Souris $m d x$} & \multirow{3}{*}{ SaCas9 } & AAV9 (IV) & \multirow{3}{*}{ Délétion de l'exon 23 du gène $D M D$} & {$[23]$} \\
\hline & & & AAV9 (IM) & & {$[24]$} \\
\hline & & & $\operatorname{AAV} 8(I M)$ & & {$[25]$} \\
\hline & & \multirow{2}{*}{ SpCas9 } & $\begin{array}{l}\text { AAV9 (IP, IM } \\
\text { ou RO) }\end{array}$ & Saut de l'exon 23 du gène $D M D$ & [19] \\
\hline & & & $\begin{array}{l}\text { Nanoparticule } \\
\text { en or (IM) }\end{array}$ & $\begin{array}{l}\text { Correction de la mutation dans le gène DMD } \\
\text { grâce à un ADN donneur }\end{array}$ & {$[8]$} \\
\hline & & SpCas9 et SaCas9 & & Délétion des exons 52 et 53 du gène $D M D$ & \\
\hline & Souris $m d x 4 c$ & SpCas9 & AAV6 (IM) & $\begin{array}{l}\text { Correction de la mutation dans l'exon } 53 \text { du } \\
\text { gène } D M D \text { grâce à un ADN donneur }\end{array}$ & [31] \\
\hline & $\begin{array}{l}\text { Myoblastes de } \\
\text { patients }\end{array}$ & SaCas9 & Plasmide & $\begin{array}{l}\text { Délétion des exons } 48 \text { à } 57 \text { pour former un } \\
\text { exon hybride } 47-58 \text { du gène } D M D\end{array}$ & [22] \\
\hline & \multirow{2}{*}{ iPS de patients } & \multirow{2}{*}{ SpCas9 } & $\begin{array}{l}\text { ARNm SpCas9 } \\
\text { et ARNg }\end{array}$ & Saut de l'exon 45 du gène $D M D$ & {$[20]$} \\
\hline & & & Plasmide & $\begin{array}{l}\text { Saut des exons } 6-7,8,43,44,45,46,50,51 \text {, } \\
52,53 \text { ou } 55 \text { du gène } D M D\end{array}$ & [21] \\
\hline \multirow{3}{*}{$\begin{array}{l}\text { Dystrophie } \\
\text { myotonique de } \\
\text { type } 1 \\
\text { (DMl) }\end{array}$} & \multirow[t]{2}{*}{ iPS de patients } & SpCas9 et SaCas9 & Plasmide & $\begin{array}{l}\text { Insertion de signaux de polyadénylation en } \\
\text { amont des répétitions CTG du gène DMPK }\end{array}$ & [29] \\
\hline & & SpCas9 & Lentivirus & Délétion de la répétition CTG du gène DMPK & {$[26]$} \\
\hline & Souris DMSXL & SaCas9 & $\operatorname{AAV}(I M)$ & Délétion de la répétition CTG du gène DMPK & [27] \\
\hline LGMD Rl & iPS de patients & SpCas9 & $\begin{array}{l}\text { Plasmide et } \\
\text { RNP Cas9 }\end{array}$ & $\begin{array}{l}\text { Correction des mutations dans le gène CAPN3 } \\
\text { grâce à un ADN donneur }\end{array}$ & [28] \\
\hline
\end{tabular}

Tableau I. Études pré-cliniques ayant utilisé le système CRISPR-Cas9 pour traiter une dystrophie musculaire.

L'avantage de ces techniques est la restauration de la protéine entière, contrairement à la thérapie par saut d'exon et par délétion d'exon que nous avons décrites. L'inconvénient de cette méthode reste la limitation de la taille de I'ADN donneur utilisable. II n'est donc pas possible de corriger de larges délétions avec cette technique.

\section{Les défis du système CRISPR}

Grâce aux progrès technologiques, de nombreuses études ont été menées afin d'explorer les bénéfices thérapeutiques du système CRISPR-Cas9, notamment pour les nombreuses dystrophies musculaires (Tableau 1). Cet outil d'édition génomique nécessite cependant de relever plusieurs défis avec en particulier le choix de l'enzyme Cas9, dont il existe plusieurs formes aux activités différentes (SaCas9, SpCas, dCas9, etc.), de l'ARN guide, ou le choix de la méthode de transport [37].

Le choix de l'enzyme Cas9 utilisée peut apporter certains avantages. La longueur de la séquence PAM diffère selon l'enzyme (3 nucléotides pour SpCas9, 5 nucléotides pour SaCas9), ce qui permet une meilleure spécificité de l'enzyme pour son substrat. II existe également des éditeurs de base qui convertissent un nucléotide du génome. Ce système utilise une déaminase fusionnée à l'enzyme nCas9 (Cas9 nickase) ou à l'enzyme dCas9 (Cas9 catalytiquement déficiente). L'avantage de cette technique est qu'elle n'induit pas de coupure de l'ADN et ne nécessite 
pas un ADN donneur pour la réparation de l'ADN. Dernièrement, Ryu et son équipe ont ainsi utilisé un éditeur de base pour substituer une guanine à une adénine, dans un modèle de souris DMD portant une mutation non-sens dans l'exon 20 du gène DMD [38]. L'enzyme dCas9, dépourvue d'activité lytique, fusionnée avec des molécules effectrices, n'implique pas le clivage de l'ADN ciblé. Cette stratégie permet d'induire l'activation ou l'inhibition de la transcription, comme pour l'utrophine (un paralogue de la dystrophine), candidat de choix à surexprimer pour réduire le phénotype DMD chez la souris $m d x$ [39].

Le transport viral du système CRISPR-Cas9 par un vecteur AAV implique plusieurs contraintes, comme la limitation de la taille d'encapsidation du virus (4.7kb), ou la présence d'anticorps spécifiques des différents sérotypes d'AAV préexistant chez la majorité des adultes à la suite d'expositions naturelles (infection) aux AAV durant l'enfance [13]. La taille d'encapsidation du virus est une contrainte majeure et dans la plupart des cas, les études menées sur des organismes modèles, utilisent deux transporteurs distincts : I'un pour l'enzyme Cas9, l'autre pour les ARN guides. Or, dans une optique thérapeutique, il est nécessaire de transporter l'enzyme Cas9 et les ARN guides dans le même vecteur afin d'augmenter l'efficacité de transport et de ciblage. Le vecteur AAV a également l'inconvénient de s'intégrer dans le génome de l'hôte traité, au niveau des ruptures occasionnées par l'enzyme Cas9 [40]. Les sites d'intégration du génome viral sont divers : région intronique $(44,7 \%)$, région intergénique $(33,4 \%)$, région exonique $(3,5 \%)$ et région régulatrice $(9,2 \%)$ et il est important de prendre en compte ces insertions dans les applications d'édition génomique. Il faut également considérer un potentiel rôle oncogène de l'AAV après son intégration, dû à la transactivation des gènes via la région ITR (inverted terminal repeats) du virus [51] $(\rightarrow)$.

$(\rightarrow)$ Voir la Synthèse de A. Rossi et A. Salvetti, $\mathrm{m} / \mathrm{s} \mathrm{n}^{\circ} 2$, février 2016 , page 167

Le choix de la méthode de transport influence également la durée d'expression du système CRISPR dans l'organisme, avec une corrélation avec la fréquence d'apparition de mutations hors cible. Une étude propose une stratégie afin de limiter l'induction de ces mutations hors cible avec l'utilisation de protéines anti-CRISPR (Acr), dont l'activité est modulée par des microARN, qui permettent de limiter l'édition génomique non souhaitée dans un type cellulaire ou un tissu spécifique [41]. Utilisant CRISPR-Cas9 et l'Acrll, qui réprime le miR122 spécifique du foie, une édition génomique spécifiquement dans le foie, et pas dans les autres tissus de l'organisme, a ainsi été obtenue.

Pour les dystrophies musculaires, l'un des défis de l'utilisation de CRISPR reste son transport ciblé au niveau du muscle et l'expression de la protéine restaurée à long terme $[42,43]$. Les résultats thérapeutiques précliniques obtenus montrent en effet souvent une expression de la protéine à long terme qui se révèle plus importante dans le muscle cardiaque que dans le muscle squelettique, ce qui s'explique par la régénération possible de ce dernier. II est donc nécessaire que le complexe CRISPR-Cas9 atteigne les cellules satellites musculaires pour éviter la répétition des injections, mais pour atteindre cet objectif, des études supplémentaires devront être entreprises.

À noter, finalement, que des études très récentes réalisée chez l’homme ont révélé la préexistence très fréquente dans le sérum, d'anticorps spécifiques de Cas9 de bactéries particulières: SaCas9 (Staphylococcus aureus Cas9) et SpCas9 (Streptococcus pyogenes Cas9), les orthologues les plus courants de Cas9 [44-46]. Ces résultats importants doivent donc être pris en considération à I'heure où les essais cliniques sont envisagés, afin de prévenir le risque éventuel lié à cette réponse immunitaire adaptative préexistante. Aucune étude ne démontre cependant que cette réponse immunitaire anti-Cas9 puisse être à l'origine d'une élimination des cellules modifiées et exprimant Cas9. L'absence d'effet de ces anticorps, qui seraient délétères pour la thérapie et limiteraient l'utilisation future chez I'homme de l'outil CRISPR-Cas9, nécessite d'être examinée et confirmée.

\section{Le système CRISPR dans la société}

Le système CRISPR est désormais envisagé comme outil thérapeutique pour l'homme (Tableau II) et plusieurs essais sont en cours d'évaluation. Aux États-Unis, la société Editas a ainsi obtenu l'autorisation de la FDA (Food and Drug Administration) pour effectuer un essai clinique chez des patients atteints d'amaurose congénitale de Leber (LCA10). D'autres essais cliniques sont également réalisés pour traiter des pathologies oncologiques, telles que le myélome ou les sarcomes, ou des pathologies hématologiques, telles que la drépanocytose et la $\beta$-thalassémie. Plusieurs défis restent néanmoins à relever avant de pouvoir utiliser cet outil comme thérapie chez l'homme. La modification du génome qu'il permet, en particulier à cause des modifications potentielles hors cible, peut aboutir à des effets adverses qu'il est aujourd'hui difficile d'anticiper. De telles mutations hors cible pourraient perturber l'ensemble de protéines interagissant entre elles et constituant un réseau. Or, nombre de ces interactions protéiques sont encore insuffisamment identifiées, et leur altération pourrait avoir des conséquences qui restent imprévisibles à long terme.

L'utilisation biaisée et/ou non encadrée de cet outil d'édition génomique pourrait conduire à des applications en dehors du strict champ thérapeutique et à des dérives liées à sa capacité de modifier des traits non pathogènes. Sans aborder les dérives eugéniques qui pourraient résulter de cet usage, la technique CRISPR-Cas9 peut être utilisée pour modifier des caractéristiques et, par exemple, augmenter chez l'animal, la production carnée ou en améliorer les qualités nutritionnelles. Outre les manipulations de $\mathrm{He}$ en Chine sur le CCR5 ( $C$-C chemokine receptor type 5 ) afin de rendre des embryons résistants à l'infection par le VIH (virus 


\begin{tabular}{|c|c|c|c|c|}
\hline Société / Sponsor & $\begin{array}{l}\text { Désigna- } \\
\text { tion }\end{array}$ & Pathologies & Méthode & $\begin{array}{l}\text { Phase de l'essai } \\
\text { clinique }\end{array}$ \\
\hline Editas & EDIT-101 & $\begin{array}{l}\text { Amaurose congéni- } \\
\text { tale de Leber (LCA10) }\end{array}$ & $\begin{array}{l}\text { Édition génomique in vivo par délétion de } \\
\text { la mutation IVS } 26 \text { présente dans le gène } \\
\text { CEP290 }\end{array}$ & Phase I/II \\
\hline $\begin{array}{l}\text { CRISPR Therapeutics et } \\
\text { Vertex Pharmaceuticals }\end{array}$ & СТХ001 & $\begin{array}{l}\text { Drépanocytose } \\
\beta \text {-thalassémie }\end{array}$ & $\begin{array}{l}\text { Édition génomique ex-vivo des cellules } \\
\text { souches hématopoïétiques par correction } \\
\text { des mutations dans le gène des } \beta \text {-globines }\end{array}$ & Phase I/II \\
\hline Université de Pennsylvanie & $H L A-A * 0201$ & $\begin{array}{l}\text { Myélome } \\
\text { Sarcome }\end{array}$ & $\begin{array}{l}\text { Édition génomique ex-vivo des lymphocytes } \\
\text { T par inhibition de l'expression du récepteur } \\
\text { TCR et de PD-1 }\end{array}$ & Phase I \\
\hline
\end{tabular}

Tableau II. Études cliniques utilisant le système CRISPR-Cas9 pour traiter des pathologies chez l'homme.

de l'immunodéficience humaine) [54] $(\rightarrow)$, il a pu être également imaginé une application humaine dans laquelle l'outil CRISPR-Cas9 pourrait être utilisé pour modifier certains gènes afin de doper génétiquement des athlètes ou des militaires [49]2. Des gènes, tels que MSTN (myostatin), EPOR (erythropoietin receptor), IGFI (insulin-like growth factor 1) ou FST (follistatin) pourraient ainsi être ciblés : par exemple, une mutation du gène MSTN provoque une hypertrophie musculaire [50]. Josiah Zayner, un chercheur en biophysique moléculaire et qui a travaillé pour la NASA, a ainsi tenté en 2017 de modifier une partie de son génome dans le but d'augmenter sa masse musculaire en s'injectant l'enzyme Cas9 et un ARN guide ciblant le gène MSTN afin d'empêcher la production de myostatine, qui inhibe la croissance musculaire. Cette expérience fut un échec notoire mais elle montre que l'outil, simple d'utilisation et peu coûteux, le rend accessible à tous scientifiques, mais aussi aux amateurs éclairés de biologie [52] $(\rightarrow)$.

Malgré son échec, Zayner a créé une société, The

$(\rightarrow)$ Voir le Repères

de M. Meyer, $m / s n^{\circ} 5$, mai 2018, page 473

$O D I N$, qui commercialise des kits permettant de réaliser de l'édition génomique à domicile, notamment en utilisant l'outil CRISPR. Cette situation a conduit les autorités fédérales et la FDA à rappeler que I'utilisation de CRISPR chez l'homme devait faire l'objet d'une autorisation, et que la vente de ces kits était illégale...

Face aux risques liés à de telles situations et à la rapidité de développement de cette technologie, en termes d'efficacité, de précision, de ciblage, de simplification et de reproductibilité, il semble donc essentiel et urgent de définir les limites de son utilisation, ses indications, et qu'un cadre soit établi par les autorités sanitaires. C'est une des conditions indispensables pour que la technologie accompagne la médecine et la science et grâce à laquelle les meilleurs bénéfices thérapeutiques seront apportés aux malades, sans risques de dérive sociétale. $\diamond$

\footnotetext{
${ }^{2}$ Le dopage génétique est défini comme un transfert d'ADN ou l'utilisation de cellules génétiquement modifiées dans le but d'améliorer une performance physique.
}

\section{SUMMARY}

\section{CRISPR-Cas9 for muscle dystrophies}

Muscular dystrophies are a group of rare muscular disorders characterized by weakness and progressive degeneration of the muscle. They are diseases of genetic origin caused by the mutation of one or more genes involved in muscle function. Despite significant progress made in the field of biotherapies in recent years, there is as yet no curative treatment available for these diseases. Studies conducted since the discovery of the CRISPR-Cas9 genomic editing tool have nevertheless led to significant and promising advances in the treatment of muscular dystrophies. CRISPR-Cas9 system allows a stable and permanent edition of the genome and should make it possible to avoid long, partially efficient and repetitive treatments. In this review, we will discuss the latest therapeutic advances obtained using the CRISPR-Cas9 system in genetic muscular dystrophies. $\diamond$

\section{REMERCIEMENTS}

Nous tenons à remercier l'ensemble des membres du laboratoire MMG et de GIPTIS.

\section{LIENS D'INTÉRÊT}

Les auteurs déclarent n'avoir aucun lien d'intérêt concernant les données publiées dans cet article.

\section{RÉFÉRENCES}

1. Frontera WR, Ochala J. Skeletal muscle: a brief review of structure and function. Calcif Tissue Int $2015 ; 96: 183-95$.

2. Carter JC, Sheehan DW, Prochoroff A, Birnkrant DJ. Muscular dystrophies. Clin Chest Med 2018 ; 39 : 377-89.

3. Al-Zaidy S, Rodino-Klapac L, Mendell JR. Gene therapy for muscular dystrophy: moving the field forward. Pediatr Neurol $2014 ; 51: 607-18$.

4. Gaj T, Gersbach CA, Barbas CF. ZFN, TALEN, and CRISPR/Cas-based methods for genome engineering. Trends Biotechnol 2013 ; 31 : 397-405.

5. Nishimasu H, Ran FA, Hsu PD, et al. Crystal structure of Cas 9 in complex with guide RNA and target DNA. Cell $2014 ; 156$ : 935-49.

6. Jiang F, Doudna JA. CRISPR-Cas9 Structures and Mechanisms. Annu Rev Biophys 2017 ; 46 : 505-29. 


\section{RÉFÉRENCES}

7. Zhang XH, Tee Ly, Wang XG, et al. Off-target effects in CRISPR/Cas9-mediated genome engineering. Mol Ther Nucleic Acids $2015 ; 4: 264$

8. Aryal NK, Wasylishen AR, Lozano G. CRISPR/Cas9 can mediate high-efficiency off-target mutations in mice in vivo. Cell Death Dis $2018 ; 9: 1099$.

9. Neldeborg S, Lin L, Stougaard M, Luo Y. Rapid and efficient gene deletion by CRISPR/Cas9. Methods Mol Biol $2019 ; 1961: 233-47$.

10. Min YL, Bassel-Duby R, Olson EN. CRISPR correction of Duchenne muscular dystrophy. Annu Rev Med $2019 ; 70: 239-55$.

11. Gee P, Xu H, Hotta A. Cellular reprogramming, genome editing, and alternative CRISPR Cas9 technologies for precise gene therapy of Duchenne muscular dystrophy. Stem Cells Int 2017 ; 8765154 .

12. Chen $G$, Abdeen $A A$, Wang $Y$, et al. A biodegradable nanocapsule delivers a Cas 9 ribonucleoprotein complex for in vivo genome editing. Nat Nanotechnol $2019 ; 14: 974-80$.

13. Lee K, Conboy M, Park HM, et al. Nanoparticle delivery of Cas 9 ribonucleoprotein and donor DNA in vivo induces homology-directed DNA repair. Nat Biomed Eng 2017 ; $1: 889-901$.

14. Lau CH, Suh Y. In vivo genome editing in animals using AAV-CRISPR system: applications to translational research of human disease. Fl000Res $2017 ; 6: 2153$

15. Nakamura K, Fujii W, Tsuboi M, et al. Generation of muscular dystrophy model rats with a CRISPR/ Cas system. Sci Rep $2014 ; 4: 5635$.

16. Amoasii L, Long C, Li H, et al. Single-cut genome editing restores dystrophin expression in a new mouse model of muscular dystrophy. Sci Transl Med 2017 ; 9.

17. Sui T, Lau YS, Liu D, et al. A novel rabbit model of Duchenne muscular dystrophy generated by CRISPR/Cas9. Dis Model Mech $2018 ; 11$.

18. Chen $\mathrm{Y}$, Zheng $\mathrm{Y}$, Kang $\mathrm{Y}$, et al. Functional disruption of the dystrophin gene in rhesus monkey using CRISPR/Cas9. Hum Mol Genet $2015 ; 24: 3764-74$

19. Demonbreun AR, Wyatt $\varepsilon$ J, Fallon KS, et al. A gene-edited mouse model of Limb-Girdle muscular dystrophy 2C for testing exon skipping. Dis Model Mech 2019 ; 13. pii: dmm040832.

20. Brennan S, Garcia-Castaneda M, Michelucci A, et al. Mouse model of severe recessive RYRIrelated myopathy. Hum Mol Genet $2019 ; 28$ : 3024-36

21. Amoasii L, Li H, Zhang Y, et al. In vivo non-invasive monitoring of dystrophin correction in a new Duchenne muscular dystrophy reporter mouse. Nat Commun $2019 ; 10: 4537$.

22. Amoasii L, Hildyard JCW, Li H, et al. Gene editing restores dystrophin expression in a canine model of Duchenne muscular dystrophy. Science $2018 ; 362: 86-91$.

23. Long C, Amoasii L, Mireault AA, et al. Postnatal genome editing partially restores dystrophin expression in a mouse model of muscular dystrophy. Science $2016 ; 351: 400-3$.

24. Ifuku M, Iwabuchi KA, Tanaka M, et al. Restoration of dystrophin protein expression by exon skipping utilizing CRISPR-Cas9 in myoblasts derived from DMD patient iPS cells. Methods Mol Biol 2018 ; 1828 : 191-217.

25. Long C, Li H, Tiburcy M, et al. Correction of diverse muscular dystrophy mutations in human engineered heart muscle by single-site genome editing. Sci Adv $2018 ; 4$ : eaap 9004.

26. Duchêne BL, Cherif K, lyombe-Engembe JP, et al. CRISPR-induced deletion with SaCas 9 restores dystrophin expression in dystrophic models in vitro and in vivo. Mol Ther $2018 ; 26: 2604-16$.

27. Nance ME, Shi R, Hakim CH, et al. AAV9 edits muscle stem cells in normal and dystrophic adult mice. Mol Ther $2019 ; 27: 1568-85$.

28. Tabebordbar M, Zhu K, Cheng JKW, et al. In vivo gene editing in dystrophic mouse muscle and muscle stem cells. Science 2016 ; 351: 407-11.

29. Nelson $\mathrm{C} \varepsilon$, Hakim CH, Ousterout DG, et al. In vivo genome editing improves muscle function in a mouse model of Duchenne muscular dystrophy. Science 2016 ; 351 : 403-7.

30. Dastidar S, Ardui S, Singh K, et la. Efficient CRISPR/Cas9-mediated editing of trinucleotide repeat expansion in myotonic dystrophy patient-derived iPS and myogenic cells. Nucleic Acids Res 2018 ; $46: 8275-98$.

31. Lo Scrudato M, Poulard K, Sourd C, et al. Genome editing of expanded CTG repeats within the human DMPK gene reduces nuclear RNA foci in muscle of DMl mice. Mol Ther $2019 ; 27: 1372-88$.
32. Selvaraj S, Dhoke NR, Kiley J, et al. Gene correction of LGMD2A patientspecific iPSCs for the development of targeted autologous cell therapy. Mol Ther $2019 ; 27: 2147-57$

33. Wang $\mathrm{Y}$, Hao L, Wang $\mathrm{H}$, et al. Therapeutic genome editing for myotonic dystrophy type 1 using CRISPR/Cas9. Mol Ther 2018; $26: 2617-30$.

34. Long C, McAnally JR, Shelton JM, et al. Prevention of muscular dystrophy in mice by CRISPR/Cas9-mediated editing of germline DNA. Science 2014 ; 345: 1184-8.

35. Bengtsson NE, Hall JK, Odom GL, et al. Muscle-specific CRISPR/Cas9 dystrophin gene editing ameliorates pathophysiology in a mouse model for Duchenne muscular dystrophy. Nat Commun 2017 ; 8 : 14454.

36. Matre PR, MuX, Wu J, et al. CRISPR/Cas9-based dystrophin restoration reveals a novel role for dystrophin in bioenergetics and stress resistance of muscle Pprogenitors. Stem Cells 2019 ; 37 : 1615-28.

37. Hwang J, Yokota T. Recent advancements in exon-skipping therapies using antisense oligonucleotides and genome editing for the treatment of various muscular dystrophies. Expert Rev Mol Med $2019 ; 21$ : e5.

38. Ryu SM, Koo T, Kim K, et al. Adenine base editing in mouse embryos and an adult mouse model of Duchenne muscular dystrophy. Nat Biotechnol 2018 ; $36: 536-9$.

39. Ricotti V, Spinty S, Roper H, et al. Safety, tolerability, and pharmacokinetics of SMT C1100, a 2-Arylbenzoxazole utrophin modulator, following singleand multiple-dose administration to pediatric patients with Duchenne muscular dystrophy. PLoS One 2016; 11 : e0152840.

40. Hanlon KS, Kleinstiver BP, Garcia SP, et al. High levels of AAV vector integration into CRISPR-induced DNA breaks. Nat Commun 2019; $10: 4439$

41. Lee J, Mou $H$, Ibraheim $R$, et al. Tissue-restricted genome editing in vivo specified by microRNA-repressible anti-CRISPR proteins. RNA $2019 ; 25$ : 1421-31.

42. Min $Y L$, Bassel-Duby R, Olson EN. CRISPR correction of Duchenne muscular dystrophy. Annu Rev Med 2019 ; 70 : 239-55.

43. Cohen J. In dogs, CRISPR fixes a muscular dystrophy. Science 2018 ; 361 : 835

44. Attenello FJ. Immunity to CRISPR-Cas9. Sci Transl Med 2019; $1: 5328$.

45. Crudele JM, Chamberlain JS. Cas 9 immunity creates challenges for CRISPR gene editing therapies. Nat Commun $2018 ; 9: 3497$.

46. Charlesworth CT, Deshpande PS, Dever DP, et al. Identification of preexisting adaptative immunity to Cas 9 proteins in humans. Nat Med $2019 ; 25: 249-54$.

49. Neuberger $\varepsilon W I$, Simon P. Gene and cell doping: the new frontier. Beyond myth or reality. Med Sport Sci 2017; 62: 91-106.

50. Schuelke M, Wagner KR, Stolz LE, et al. Myostatin mutation associated with gross muscle hypertrophy in a child. $N$ Engl J Med $2004 ; 350$ : 2682-8.

51. Rossi $A$, Salvetti $A$. Intégration des vecteurs $A A V$ et mutagenèse insertionnelle. Med Sci (Paris) $2016 ; 32: 167-74$

52. Meyer M. Biologie et médecine «do-it-yourself ». Histoire, pratiques, enjeux. Med Sci (Paris) 2018 ; $34: 473-9$.

53. Smithies 0 , Gregg RG, Boggs SS, et al. Insertion of DNA sequences into the human chromosomal beta-globin locus by homologous recombination. Nature $1985 ; 317: 230-4$

54. Jordan B. Bébés CRISPR : anatomie d'une transgression. Med Sci (Paris) $2019 ; 35: 266-70$

TIRÉS À PART

N. Levy

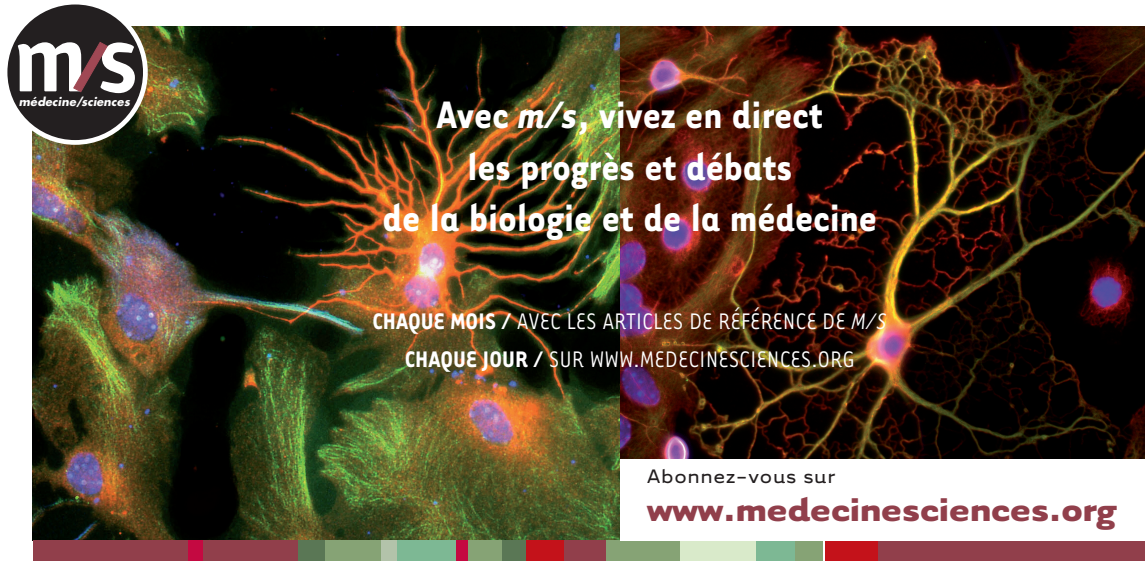

